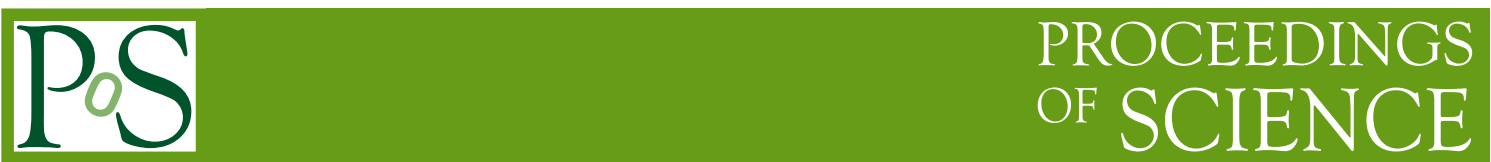

\title{
Observations of blazars by the ESA INTEGRAL satellite
}

\author{
Rene Hudec*t \\ Astronomical Institute of the Academy of Sciences of the Czech Republic \\ E-mail: rene.hudec@gmail.com
}

\section{Filip Munz}

Astronomical Institute of the Academy of Sciences of the Czech Republic

E-mail: munz@physics.muni.cz

\section{Elena Pian}

INAF, Trieste, Italy

E-mail: pian@oats.inaf.it

We refer on analysis of the ESA INTEGRAL satellite data for specific class of active galactic nuclei - blazars. These objects represent promising sources to be observed by INTEGRAL, especially during their active states. Suitable strategy for the future analysis is proposed and discussed.

Workshop on Blazar Variability across the Electromagnetic Spectrum

April 22-25, 2008

Palaiseau, France

* Speaker.

${ }^{\dagger}$ A footnote may follow. 


\section{Introduction}

Blazars represent the most extreme class of active galaxies, they are powerful and variable. They are observed in all wavelength bands - from radio through VHE gamma frequencies, with maximum spectral output and largest variability often at gamma ray energies. 66 blazars were identified as sources of $\geq 100 \mathrm{MeV}$ emission by EGRET aboard CGRO (Hartman, [1999]) and 6 blazars were identified as VHE gamma sources $(\geq 350 \mathrm{GeV})$ by Cerenkov telescopes (Krawczynski, [2004]). It is obvious that blazars represent suitable targets for INTEGRAL satellite (Winkler et al., [2003]) especially during active states (flares).

\section{INTEGRAL observations}

The INTEGRAL observations are basically divided into the following categories: (i) AO-1,2,3 Program (allocated pointed observations), (ii) Core Program CP (Galactic Plane Scans, Galactic Center Deep Exposure,...), and (iii) Objects inside FOV of AO-1,2,3 observations.

\section{Core Programme Observations}

Blazars in the INTEGRAL Galactic Plane Scans (GPS) represent a promising group of objects for the study within the INTEGRAL CP. The GPS zone is usually neglected by extragalactic astronomers due to heavy obscuration: in optical, $\sim 20 \%$ of the sky is obscured by our Galaxy, while the gamma-ray telescopes on board INTEGRAL allow detectability of up to few mCrabs in the most exposed GPS regions.

Seven optically bright (with $\mathrm{V} \leq 17 \mathrm{mag}$, to be detected by the INTEGRAL OMC camera) blazars were identified within galactic scans of INTEGRAL, namely: 1ES 0647+250, PKS 0823223 (no gamma from EGRET, grav. lensing candidate), 1ES 2344+514 (TeV gamma ray source, very close), 8C 0149+710 (BL Lac candidate?), 4C 47.08, 87GB 02109+5130 (poorly understood blazar, $\mathrm{TeV}$ candidate), and BL Lac (the prototype).

While the prototype object BL Lac is well studied, most of the INTEGRAL GPS blazars are poorly investigated and poorly understood so far. The study with Sonneberg Observatory Archival Plates reveals that most of these objects are optically variable, hence a gamma ray variability can be expected.

Below the detection limit of the INTEGRAL OMC on board camera is blazar NRAO530 (1730-130), which is an example of blazar with violent optical activity (4 mag within 1 month). In flare, the object is expected to be much brighter also in gamma. This strengthens the role of optical monitoring and ToO program - the flare can be recognized by optical monitoring with small (D $\sim 50 \mathrm{~cm}$ ) telescopes.

All the above mentioned blazars in INTEGRAL GPS have been investigated with INTEGRAL $\mathrm{CP}$ data (IBIS and JEM-X telescopes). We have developed method for data mining in archival data for faint sources which has resulted in several positive detection by high energy instruments on board INTEGRAL. For other targets, the quiet level is still below the sensitivity threshold of the instruments. However, the positive detection may be possible in the future as (i) there will be more cumulative time available and (ii) the probability to see a blazar during a flare (and hence much brighter) will also increase with time. 


\section{AO Observations}

Additional blazars have been identified in the fields represented by the AO-1 and AO-2 observations of other scheduled targets, covered by up to $400 \mathrm{ksec}$ cumulative exposure time. The analysis of these objects are in progress, as the data are becoming public.

Regarding the pointed observations of blazars by INTEGRAL, the AO-2 ToO blazar observation No. 220049 by Pian et al. ([2005]) has provided promising results. This collaborative proposal was based on extended optical and/or X-ray monitoring (RXTE ASM and others) of flaring activity of a large list of blazars and, alternatively, on soft gamma-ray monitoring by INTEGRAL itself (serendipitous detection of a flaring blazar in the IBIS FOV). Then ToO INTEGRAL observation was activated meeting the trigger criteria (major flaring event). The program was coordinated with the XMM Newton ToO program.

Blazar S5 0716+714 was the target of this ToO observation. This is a BL Lac object, intensively monitored at radio and optical wavelengths by Whole Earth Blazar Telescope (more than 40 telescopes, Villata et al.,[2004]). The ToO was triggered by optical activity - 2 outbursts up to the extreme level of $R=12.1 \mathrm{mag}$ (historical maximum, light increase by 1 mag in 2 weeks and 2 magnitudes in 4 months) and, consequently, the INTEGRAL ToO observation was performed in the time interval 2004 April 2-7 (Pian et al.,[2005]).

The object was observed at somewhat higher (2x) gamma-ray state when in Oct 2000 (BeppoSAX ToO, Tagliaferri et al., [2003]) $(\mathrm{R}=12.5$ versus 12.1$)$. There was a low signal/noise ratio and hence no intra-orbit variability study was possible, and no spectrum extraction. Within the INTEGRAL observation, the S5 0716+714 was detected only by IBIS ISGRI at 4.5 sigma, in 30$60 \mathrm{keV}$ band, for a count rate of 0.11 counts/s (exposure $280 \mathrm{ksec}$ ). No signal above $60 \mathrm{keV}$ was detected. The target was better detected at the beginning (decline), hence the useful exposure was reduced to $84 \mathrm{ksec}$. There was no positive detection in IBIS/PICsIT, SPI and JEM-X (less than 292, 6 and 6 mCrab, Pian et al., [2005]).

Within the ToO observation, another extragalactic sources were observed in the IBIS FOV (19 x 19 deg at half response) - 3 additional AGNs with higher significance than the main blazar target. These 3 AGNs were observed up to $100 \mathrm{keV}$ with no intra-orbit variability study possible. However, the spectrum extraction was possible for S5 0836+710 (high z blazar of the FSRQ subclass): single power-law spectrum, for Mkn 6 (bright Seyfert): single power law spectrum, and for Mkn 3 (bright Seyfert): broken power-law with cutoff at $\geq 50 \mathrm{keV}$ (Pian et al., [2005]).

This AO-2 blazar observation clearly confirmed that even with relatively short exposures, the INTEGRAL is an efficient tool to study bright AGNs at high Galactic latitudes and also has demonstrated the importance of high-energy instruments with large FOV and good angular resolution (like IBIS/INTEGRAL). In addition, the detection of two high $\mathrm{z}$ blazars (S5 0836+710 at $\mathrm{z}=2.17$ and PKS 1830-21 at $\mathrm{z}=2.51$ ), the most distant objects seen by INTEGRAL so far shows that INTEGRAL can also play a role in investigation of high $\mathrm{z}$ Universe.

Very recently, an INTEGRAL AO-3 ToO observation of 3C454.3 ( $\mathrm{z}=0.859$ ) was performed, with preliminary results given by L. Foschini et al. ([2005], PI E. Pian with a large collaboration). This ToO was triggered by high optical (T. Balonek, VSNET alert) and X-ray (BAT Swift) activity of the source. The INTEGRAL observation started 2005 May 15, at 18:40 UT, with exposure of 200 ksec. The source was clearly detected by IBIS/ISGRI in the 20-40 and 40-100 keV energy bands, 
Figure 1: The IBIS total exposure times for blazars, CP data, revolutions 1-250.

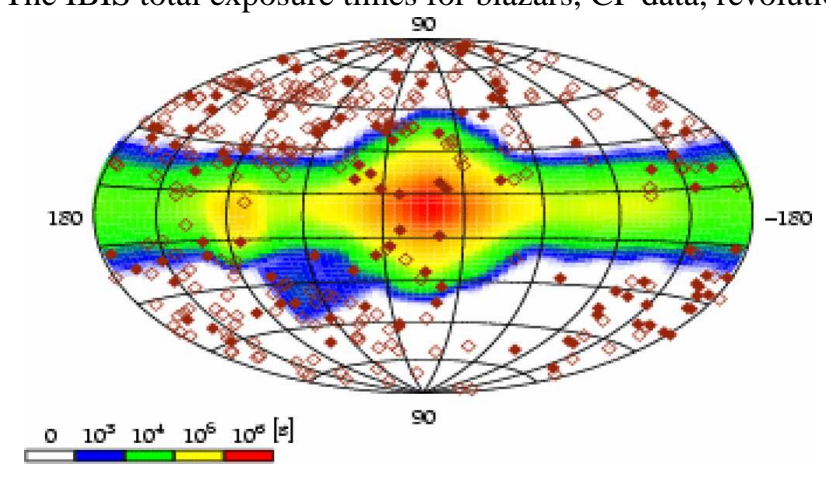

with a significance of 20 and 15 sigma. The observed fluxes were $1.02 \pm 0.05 \mathrm{cts} / \mathrm{s}=9.4 \pm 0.5$ $\mathrm{mCrab}$ in $20-40 \mathrm{keV}, 1.00 \pm 0.08 \mathrm{cts} / \mathrm{s}=13 \pm \mathrm{mCrab}$ in $40-100 \mathrm{keV}$, and $1.6 \times 10^{-10} \mathrm{ergcm}^{-2} \mathrm{~s}^{-1}$ in 20-100 keV band. The observed spectrum was flat, with photon index $2.2 \pm 0.2$ and normalization $0.13(+0.10-0.06) \mathrm{phcm}^{-2} \mathrm{~s}^{-1} \mathrm{keV}^{-1}$. The allocated $200 \mathrm{ks}$ were however not enough to detect the object up to $400 \mathrm{keV}$, more than $400 \mathrm{ks}$ would be necessary for that. It is important to continue the future INTEGRAL AO observations of blazars with longer exposures in order to fully show the importance of scientific study of blazars with this satellite.

\section{Conclusion}

For blazars in GPS, few positive detections by high-energy instruments on board INTEGRAL have been confirmed by method of data mining for faint sources, with more expected in the future. For remaining targets, the quiet level is still below the sensitivity threshold of the instruments. On the other hand, the positive detection may be possible in the future as: (i) there will be more cumulative time available, and (ii) the probability to see a blazar during a flare (and hence much brighter) will also increase with time.

For the AO blazar observation, it has been confirmed that with relatively short exposures the INTEGRAL is efficient tool to study bright AGNs at high Galactic latitudes. This also confirms the importance of high-energy instruments with large FOV and good angular resolution. Furthermore, it is also an excellent proof that the approach by Pian et al. is the right one for variable sources and INTEGRAL - extended monitoring and ToO observations in active states. Based on these results, we suggest to recognize the scientific value of ToO observations of blazars (and highly variable objects in general)by the INTEGRAL satellite.

\section{Acknowledgement}

We acknowledge the support provided by the ESA PECS Projec 98023. Some parts of this study are linked to the project 205/08/1207 by the Grant Agency of the Czech Republic. 
Figure 2: The IBIS light curve for BL Lac. The spectral changes of the source are obvious (left). The composition of INTEGRAL public data used (right)
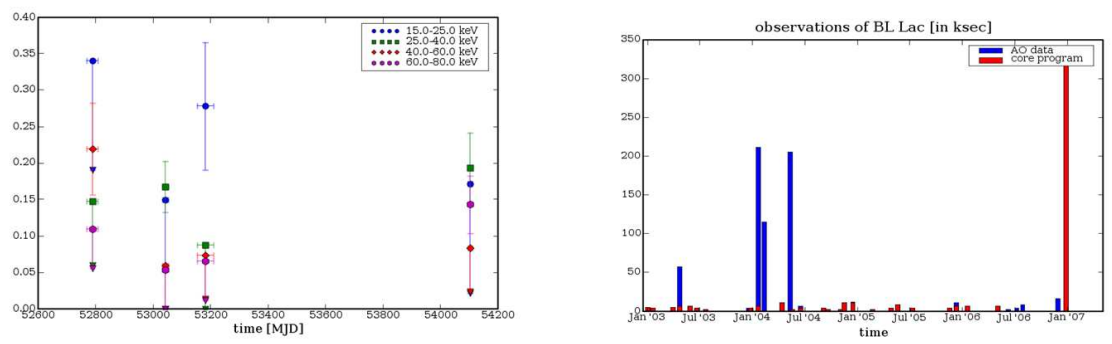

Figure 3: The IBIS mosaic images for BL Lac for two different time intervals. The spectral changes of the source are obvious. Top: MJD interval 54085.82-54113.01 i.e. 27.19 days, size 88E120 [pixels $0.082[\mathrm{E} 0.082] \mathrm{U}$ U exposure $280.888 \mathrm{ksec}, 15.0-25.0 \mathrm{keV}$ : no peak - upper limit 0.086ct/s, 25.0-40.0 keV : peak $0.193+-0.0484 \mathrm{ct} / \mathrm{s}$. Bottom: MJD interval 52985.44-53045.66 i.e. 60.22 days, size 88E120 [pixels $0.082[\mathrm{E} 0.082]$ Ů exposure $459.019 \mathrm{kses}, 15.0-25.0 \mathrm{keV}$ : peak $0.278+-0.0874 \mathrm{ct} / \mathrm{s}, 25.0-40.0 \mathrm{keV}$ : no peak - upper limit $0.088 \mathrm{ct} / \mathrm{s}$
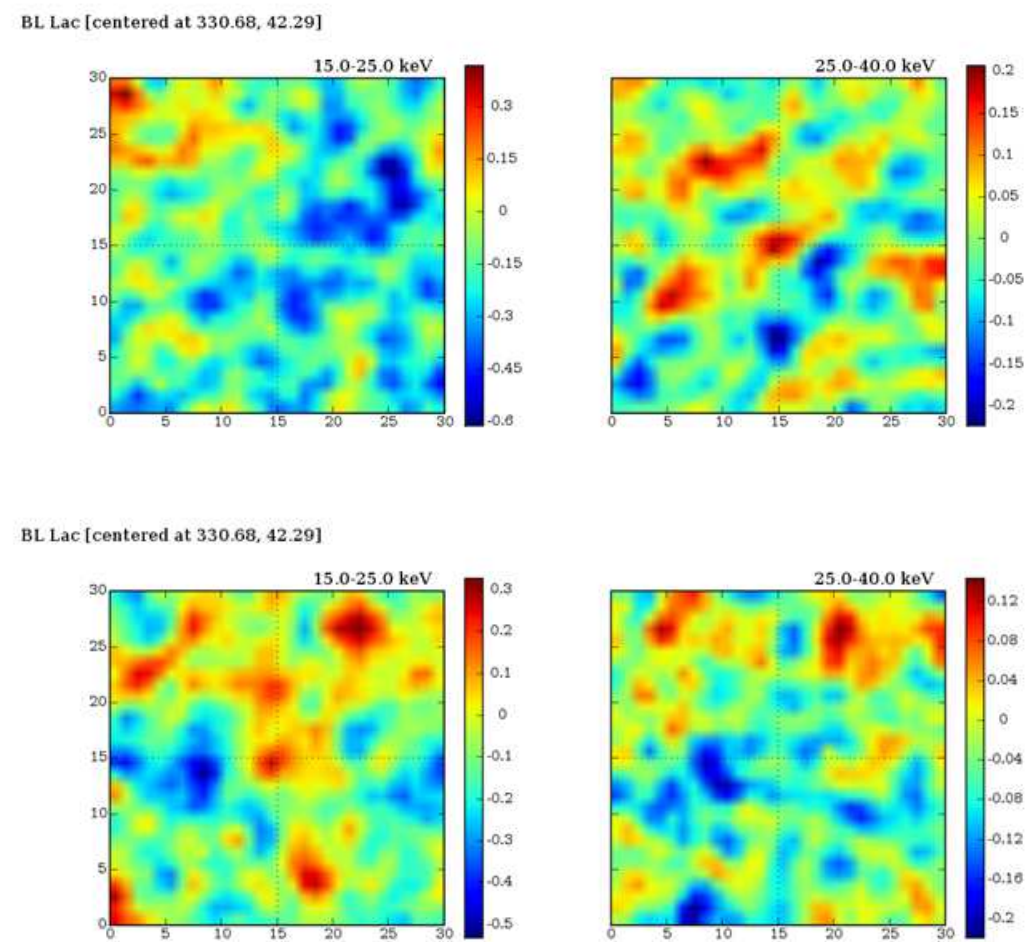
Figure 4: Left: Historical optical light curve of NRAO530. The source exhibits rare but large amplitude optical flares (4 mag). Right: Optical monitoring of GPS blazar 4C47.08 (Tosti et al., 2005).
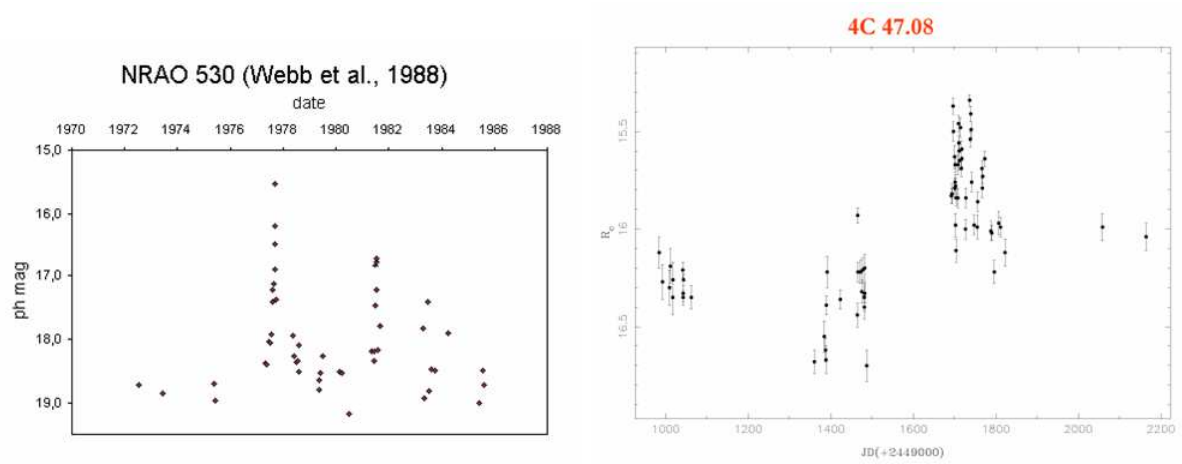

Figure 5: IBIS total exposure times for blazars, all data, revolution 1-250

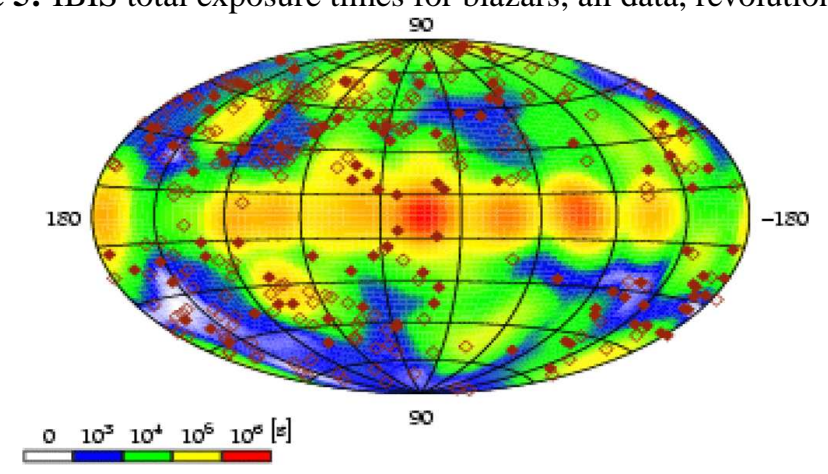

Figure 6: The optical light curve of $3 \mathrm{C} 454.3$ around the time of the INTEGRAL AO-3 ToO observation

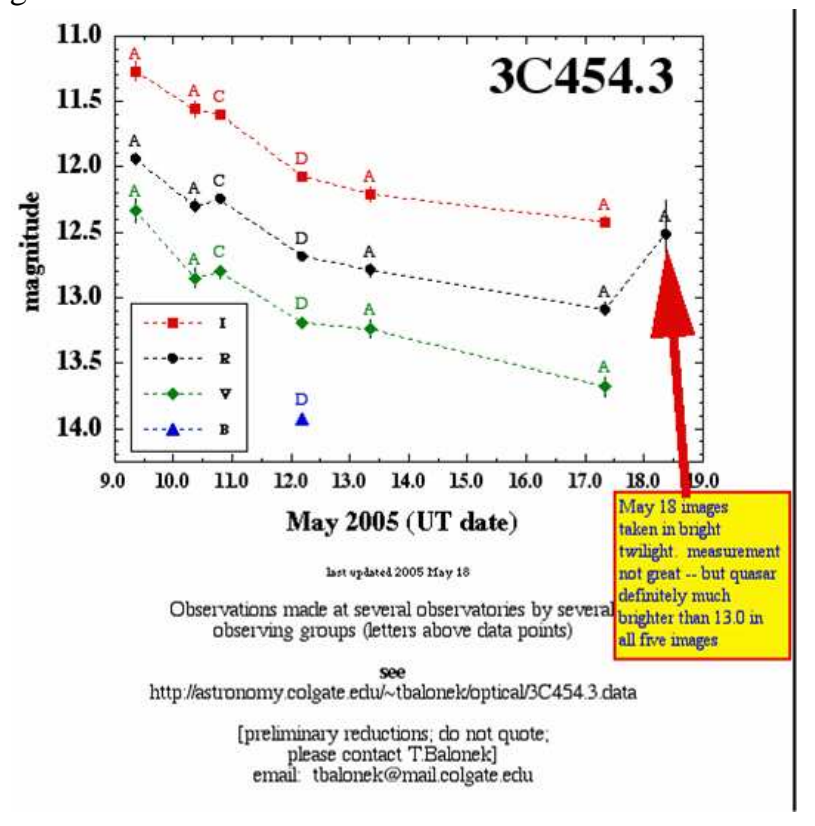


Figure 7: INTEGRAL IBIS/ISGRI images of 3C454.3, 20-40 (left) and 100-200 keV (right)
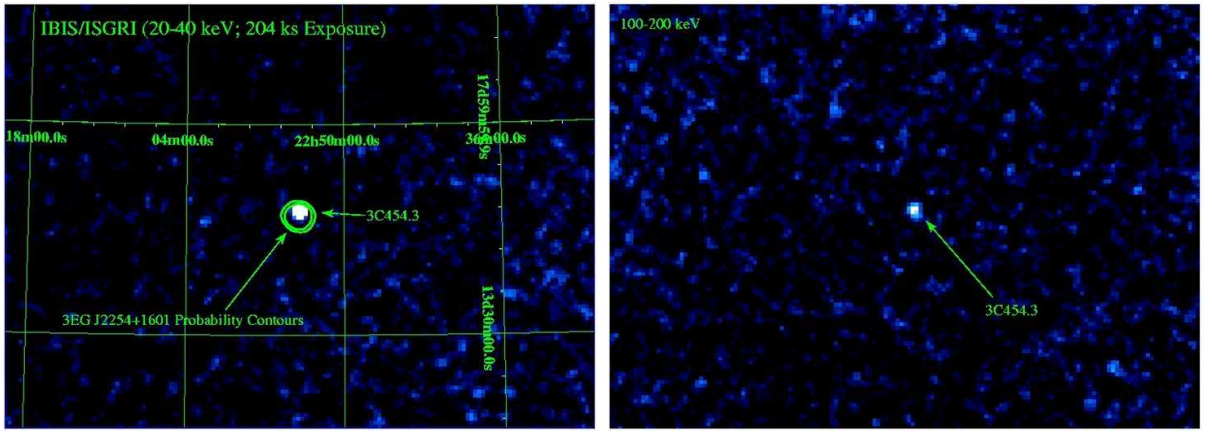

Figure 8: The most significant result of the procedure of searches for faint sources. The flux corresponding to the excess in lower spectral band for Mrk 501 is (1.57 ś 0.24$) 10-11 \mathrm{erg} / \mathrm{cm} 2 / \mathrm{s}$. The coordinates of the images are given in pixels, one pixel being 4.9 arcmin; mosaics are centered on the catalogue position of the source.
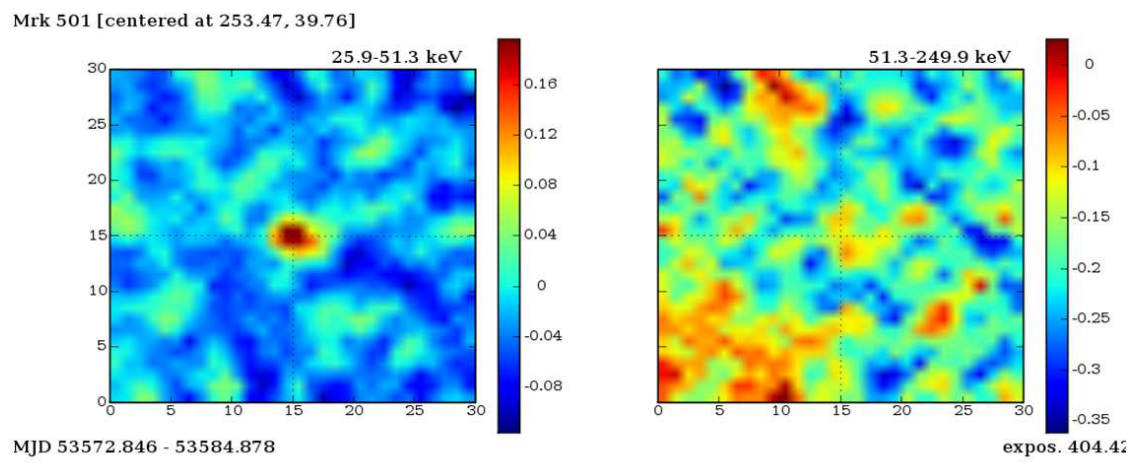

MJD 53572.846 - 53584.878

\section{References}

[2005] Foschini, L. et al., 2005, ATEL 497.

[1999] Hartman, R. et al., 1999, ApJSS, 123, 79.

[2004] Krawczynski, H., 2004, New Astronomy Reviews, 48, 367.

[2005] Pian, E.; Foschini, L.; Beckmann, V.; Sillanpää, A.; Soldi, S.; Tagliaferri, G.; Takalo, L.; Barr, P.; Ghisellini, G.; Malaguti, G.; Maraschi, L.; Palumbo, G. G. C.; Treves, A.; Courvoisier, T. J.-L.; Di Cocco, G.; Gehrels, N.; Giommi, P.; Hudec, R.; Lindfors, E.; Marcowith, A.; Nilsson, K.; Pasanen, M.; Pursimo, T.; Raiteri, C. M.; Savolainen, T.; Sikora, M.; Tornikoski, M.; Tosti, G.; Türler, M.;

Valtaoja, E.; Villata, M.; Walter, R., 2005, A\&A 429, 427.

[2003] Tagliaferri, G.; Ravasio, M.; Ghisellini, G.; Giommi, P.; Massaro, E.; Nesci, R.; Tosti, G.; Aller, M. F.; Aller, H. D.; Celotti, A.; Maraschi, L.; Tavecchio, F.; Wolter, A., 2003, A\&A 400, 477.

[2005] Tosti, G. et al., 2005, http://astro.fisica.unipg.it/PGblazar/tabella2000.htm

[2004] Villata, M. et al., 2004, A\&A, 424, 497.

[1988] Webb J. R. et al., 1988, AJ 95, 374.

[2003] Winkler, C. et al., 2003, A\&A, 411, 7. 